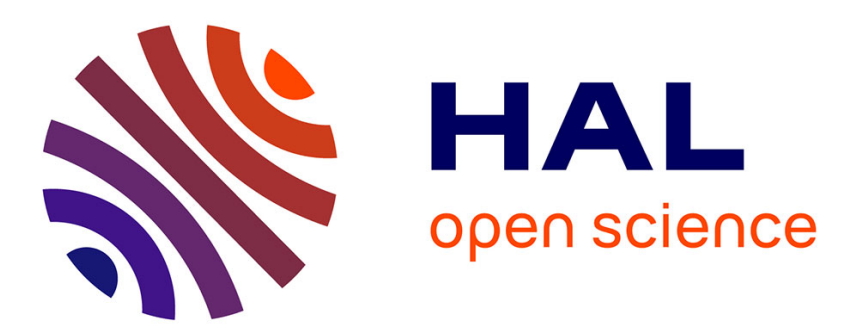

\title{
Improvement of Human-Plant Interactivity via Industrial Cloud-Based Supervisory Control and Data Acquisition System
}

Tomáš Lojka, Iveta Zolotová

\section{- To cite this version:}

Tomáš Lojka, Iveta Zolotová. Improvement of Human-Plant Interactivity via Industrial Cloud-Based Supervisory Control and Data Acquisition System. IFIP International Conference on Advances in Production Management Systems (APMS), Sep 2014, Ajaccio, France. pp.83-90, 10.1007/978-3-66244733-8_11. hal-01387152

\section{HAL Id: hal-01387152 \\ https://inria.hal.science/hal-01387152}

Submitted on 25 Oct 2016

HAL is a multi-disciplinary open access archive for the deposit and dissemination of scientific research documents, whether they are published or not. The documents may come from teaching and research institutions in France or abroad, or from public or private research centers.
L'archive ouverte pluridisciplinaire HAL, est destinée au dépôt et à la diffusion de documents scientifiques de niveau recherche, publiés ou non, émanant des établissements d'enseignement et de recherche français ou étrangers, des laboratoires publics ou privés.

\section{(c)(1)}

Distributed under a Creative Commons Attribution| 4.0 International License 


\title{
Improvement of Human-plant Interactivity via Industrial Cloud-based Supervisory Control and Data Acquisition System
}

\author{
Tomáš Lojka and Iveta Zolotová \\ Department of Cybernetics and Artificial intelligence, Košice, Slovakia. \\ \{tomas.lojka, iveta.zolotova\}@tuke.sk
}

\begin{abstract}
Industrial companies look for the best way to be perfectly optimized, failure resistant, how to handle increasing amounts of information, and have more open and reliable union with their customers and suppliers. This paper focuses on the improvement of industry performance through the integration of SCADA/HMI (Supervisory Control and Data Acquisition/Human Machine Interface), SOA (Serviced Oriented Architecture) and cloud computing. This paper's contribution is in a failover and high availability solution for small and medium industry companies. The contribution is based on the integration of the cloud and SCADA/HMI, and contains a developed SOA load balancer for better accessibility to the cloud. This whole solution improves performance, reliability and availability. It is cost-effective and includes a mobile device implementation for controlling and monitoring systems.
\end{abstract}

Keywords: Cloud manufacturing, database systems, data storage systems, network-attached storage, SCADA/HMI systems, SOA.

\section{Introduction}

Industrial companies intend to reduce their operating costs and expenses. Therefore, they need solutions that will help them have full, real-time control over an entire plant [1]. The needed features of such solutions are stability, flexibility, fault-tolerance, high accessible storage of information and good connectivity. This will make every internal and external process more effective, highly available, and cost-effective. The communication interactivity and complexity will be enlarged.

One of the recent technological waves, cloud computing, fulfils industrial companies' needs [2]. Cloud computing brings the option of integration a SCADA/HMI and the cloud [3]. The integration can reduce industrial companies' costs, increase reliability, security and system stability with fail tolerance to industrial companies' systems errors [1]. This leads to "smart" industrial companies like homes in IBM vision [4]. In SDACA/HMI and cloud implantation, local parts of an industrial company's ICS (Information and Control System) are moved out to the cloud. The SCADA/HMI and cloud integration also brings a solution to the increasing demands for the amount of quick, easy and secured access to the information inside industrial companies, where

adfa, p. 1, 2011.

(C) Springer-Verlag Berlin Heidelberg 2011 
information is important not only for critical control. The amount of information in an ICS increases every year and with new parts in the ICS. Industrial companies struggle with processing and saving large amounts of data and ensuring the security of manufacture information. Industrial companies easily overcome demands of increasing amount of information with hosting a SCADA/HMI (2) in the cloud with using cloud benefits (2) and risks (2.1).

We defined our SCADA/HMI cloud-based solution (3) with a failover SOA (Service Oriented Architecture) and a failover MySQL cluster (4) to reach improvements in performance, reliability and availability, and to find a cost-effective solution with the involved mobile device implementation for controlling and monitoring systems. The solution is aimed at small industrial companies which want to manage their own cloud. This solution was created to fulfil small industrial companies' requirements in a costefficient way [5]. Moreover, this solution is open enough to supporters and customers, but not publicly open to the internet. In this solution, we chose a SOA and cloud (private, realized with VMware). Service-oriented architecture is also used for communication with mobile clients, and separated load balancer service.

\section{Hosting a SCADA/HMI in the cloud}

There are many options how to implement a SCADA/HMI system in the cloud to get all benefits from a cloud-based SCADA/HMI system.

A cloud-based SCADA/HMI system provides robust remote access and independency of the client platform. We identified three option how to host a SCADA/HMI in the cloud to reach best contribution for our implementation:

- Outside cloud-based SCADA/HMI is situated outside of the cloud, SCADA/HMI is connected to the cloud. Data is transmitted to the cloud and classified [3]. Main purpose is data analysis and easy access to data. Data is mainly stored in the cloud to be always available and secure. Additional reporting and analysis services are implemented in the cloud to increase the efficiency of data acquisition and historization. Clients retrieve data from the cloud [3]

- Inside cloud-based SCADA/HMI is running in the cloud and it is connected to the technological layer [2]. In this solution, cloud applications are remotely connected to the technological layer. Commands are sent to the technological layer and then processed. Data is polled from the technological layer and remotely sent to the cloud.

- Inside/Outside cloud-based SCADA/HMI is primarily running outside of the cloud, while some SCADA/HMI application runs in the cloud as well.

In our implementation, we focused on the inside cloud-based SCADA/HMI, which is depicted in Fig. 1. 


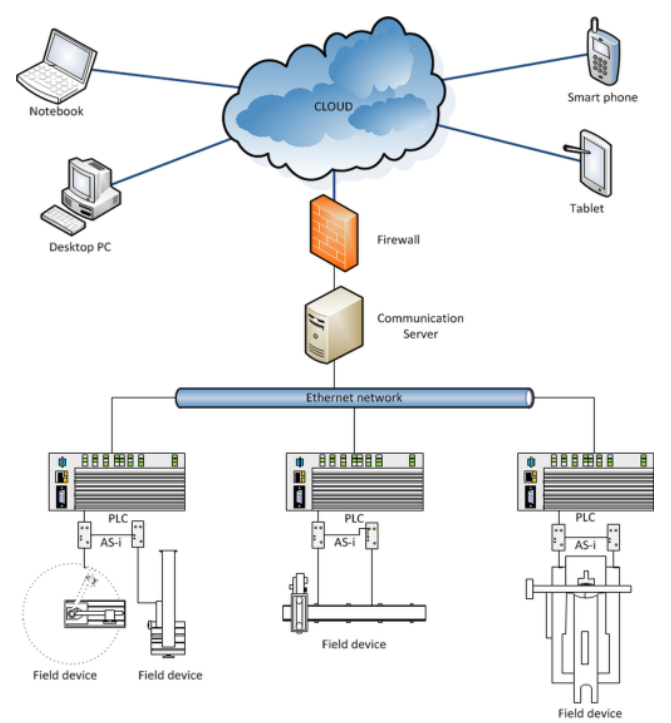

Fig. 1. Inside cloud-based SCADA/HMI system with the technological layer, communication server and firewall to protect the access to the technological layer. The SCADA/HMI application runs in the cloud and the cloud clients are connected to the application.

Our purpose was to integrate the benefits of the cloud, such as high availability, virtualization, load balancing, uptime etc. Integration provides multi-use remote access where the SCADA/HMI system is guest in the cloud environment. Hosting a SCADA/HMI in the cloud allows a user to track data with various hardware platforms (smartphones, tablets, notebook and so on).

Big benefit of a cloud-based SCADA/HMI solution is data accessibility. This accessibility ensures stable access to data. Data informational content in ICS is important for decreasing the risks of bad decisions due to no or insufficiently information.

The cloud supports sharing information and increases the speed of accessing manufacture data, also for customers. For example, if a customer (another industrial company) urgently needs to change their production and needs raw materials with other quality, then the industrial company can easily change the requirements of product quality in the supplier's industrial company.

An ICS has internal or external suppliers which maintain, explore and upgrade the ICS. In such situations, it is beneficial for suppliers to have a distributed ICS solution in the cloud due the increased complexity between the supplier and customer.

Another example, the supplier of conveyers can easily reach data about conveyers, analyse them, predict and prevent failures and decrease the downtime in production.

Implementing a SCADA/HMI in the cloud means reaching benefits such as optimization, flexibility, scalability, availability and better cost-efficiency [2].

On the other hand, connection of the cloud and a SCADA/HMI has negatives as well. These are described in sub-section 2.1. 


\subsection{Risks of a cloud-based SCADA/HMI}

We identified the risks of a cloud base SCADA/HMI implantation. First, the cloud makes a SCADA/HMI system more open. Commands and data in the cloud can be sniffed, modified, spoofed or lost during the communication. Moreover, the connection of a SCADA/HMI and the cloud opens a door into the ICS. The attackers then can access the technological and the SCADA/HMI layer of any ICS.

Integrating a SCADA/HMI into the cloud also has the same risks like a typical cloud realization. Data might be accessible with the same service with different security access for different industrial companies or humans. More specifically, another client has access to the same cloud, and the data is only internally separated. There might be a potential risk. Attackers can find and abuse services or applications running in the cloud.

Applications which run in the cloud might also have direct access to the ICS technological layer. In such a case, it is important to secure the ICS communication, because some protocols in automation are not secured. SCADA/HMI systems regularly use MODBUS/TCP, DNP3, IEC-140 for control and automation [1]. Some protocols lack protection [6]. Industrial companies with some insecure communication protocols are more prone to attacks (man-in-the-middle (MiTM) attacks) [7].

The cloud offers big benefits for a SCADA/HMI or other layers of the ICS, but its security might not be reliable. The data is not the only thing that is supposed to be encrypted and secured inside the cloud. The connection to the technological layer and other parts of any ICS, which are not usually part of the cloud, require security measures as well. A simple solution for security is separating the data. Data which is important to suppliers or customers might be secured and placed in the cloud. Direct connection to the technological layer is risky. An alternative is creating a hybrid cloud. Another solution might be a gateway to field devices with a special protocol. This gateway creates two separated layers. The first one is for an internal SCADA/HMI part and the other layer is used for the cloud SCADA/HMI part [1].

\section{Analysis of the cloud-based SCADA/HMI Solution and Connectivity in Laboratory Conditions}

We implemented an inside cloud-based SCADA/HMI solution using a private cloud and the ICS with the SCADA/HMI system from Wonderware and Rockwell Automation [8]. We extended the ICS with cloud benefits, to share information from the SCADA/HMI and MES (Manufacturing Execution Systems) layer and used it in education exercises. Clients can then work with data anywhere and extend their own SCADA/HMI solution with cloud stored data from real technology process. Technological process is without risks and the connection to the field devices is limited.

Also, we consider the option if someone unauthorized gets access to the cloud stored data or some students try to do something unauthorized.

To design a cost-effective solution for a small company, and a solution with closed technological, layers we chose to use a private cloud. Another reason was the situation 
when providers do some changes in their security or service functionality. The SCADA/HMI and another layer of the ICS might be sensitive to such changes, which might endanger processes in the industrial company (for example slowing down query execution). We used our own private cloud solution and with OpenVPN (Open Virtual Private Net) access (for external customers and suppliers) to have better security, be more flexible in customization and see all needed sources for realization.

In our solution for the SCADA/HMI and the cloud implementation, we firstly created a storage place. We implemented a NAS (Network Attached Storage) with a CIFS (Common Internet File System) [9]. A NAS is a storage device for VMware virtual machines. This implementation enables HA (High Availability) and gives independence to the SCADA/HMI application from the hardware implementation on the server side. If the physical server fails, another server will be used to continue processing data. Data is not bound to the server but to the data storage. In our solution, we implemented an outside SCADA/HMI cloud solution. This solution can be set in a small industrial company which needs to share large amount of data to their customers, suppliers or internal workers. The solution will be used to share data from the SCADA/HMI layer.

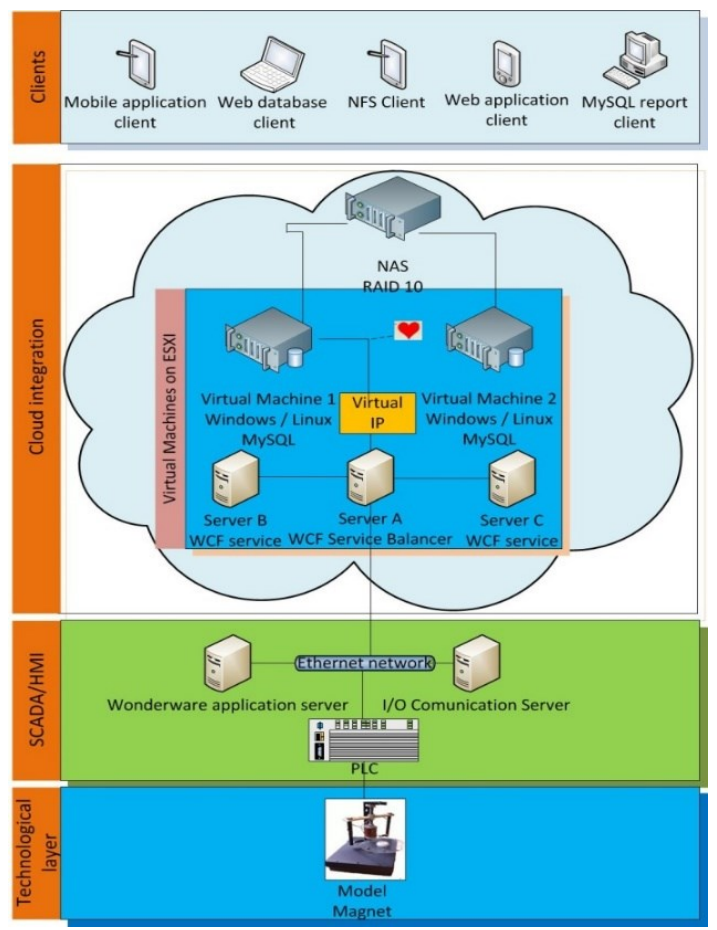

Fig. 2. Improvement of Human-plant Interactivity with better cloud accessibility.

Data storage systems should meet selected requirements (high availability, reliability and others) [2]. These requirements might be in conflict. For example, availability, scalability and data consistency might be in conflict $[1,2]$. In our solution, we used MySQL Cluster [11], where we gave more importance to data availability [10]. 
Not only storing data is important, but also the infrastructure for accessing it. Because we chose the implementation of a private cloud in our experiment, we implemented a service oriented architecture (SOA) in our architecture with a service that retrieves data from the communication server. The communication server communicates with the technological layer of our ICS. The service is also used to saving data to the cloud storage and its retrieval. The service communicates with HMI clients.

\section{$4 \quad$ Realised Solution in Laboratory}

We created and tested solution, in which data is accessible to every client. We chose the database to store data from technological process, but we also chose NFS due to its advantage of quick saving of reports in the cloud. For users are the documents accessible from NAS with the utilities which NAS has. Data is available through MySQL servers, which were realized on virtual machines on an ESXI server from VMware.

Our solution is extended with an application server. The application server is intended to communicate with the technological process, HMI clients and data storage.

The technological layer consists of a laboratory controlled model. The model is controlled by a PLC (Programmable Logic Controller). The application server communicates with the PLC through an OPC server. Data is cached in the application server. If the value of same data changes, a new event occurs. The application server writes (updates/inserts) the data to the database placed behind the application server in the event. The application server hosts a WCF (Windows Communication Foundation) service. To satisfy the failover requirement, we created a SOA load balancer. This balancer sends client requests to the service. If the balancer fails to communicate with the service or has insufficient low response from the service, it transmits the client request to another service. The balancer has memory where it saves a request after it was successfully sent. In our solution, we used service-oriented architecture (SOA) which is connected to the technological layer and to the data storage and also to the HMI clients. Inside the service, there is an implemented connection to the MySQL database and the HMI clients.

The MySQL database is placed on two independent virtual machines, which are clustered with the storage NAS. It is configured to use raid 10. Both virtual machines have their own MySQL instance and are connected together with a heartbeat connection [11].

These servers have one virtual IP address, which is used by the application server and MySQL clients (for example, MySQL report client). Virtual machines run Windows Server 2008 R2, but Linux can be used as well. Data is secured with MySQL security. MySQL data is accessible from every place inside the private network. The MySQL database stores data from the technological layer and critical controls commands from the HMI layer. This data is accessible for every SCADA/HMI application on a device or a PC.

MySQL client is not supported by many SCADA/HMI systems. However, there are libraries which can be implemented to allow the use of MySQL. The access to data ensures tolerance to faults, which is guaranteed with ours created cluster and the heartbeat connection of MySQL [11]. 


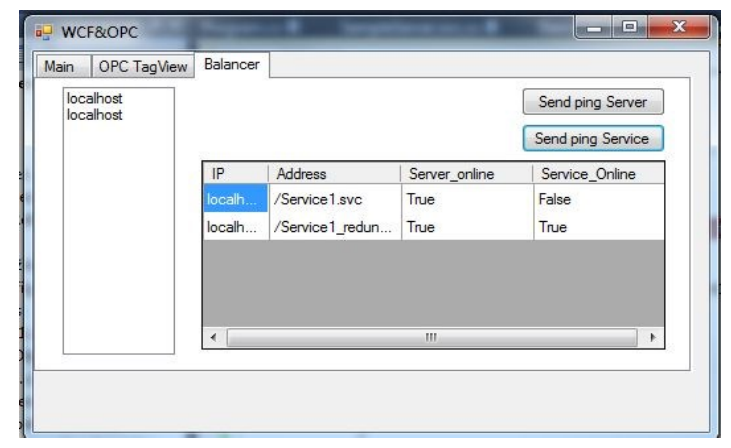

Fig. 3. Graphical interpretation of the SOA load balancer

The HMI clients can connect to the service through an installed application or through a website. These clients are used like mobile HMI applications for manufacture operators.

Clients can connect to the NAS (for example, with a web browser) and access files. Clients can use FTP, NFS and SMD protocols. These clients are mainly oriented for downloading created reports, saving and sharing business documents.

The last client type is a client application for a direct connection to the MySQL service.

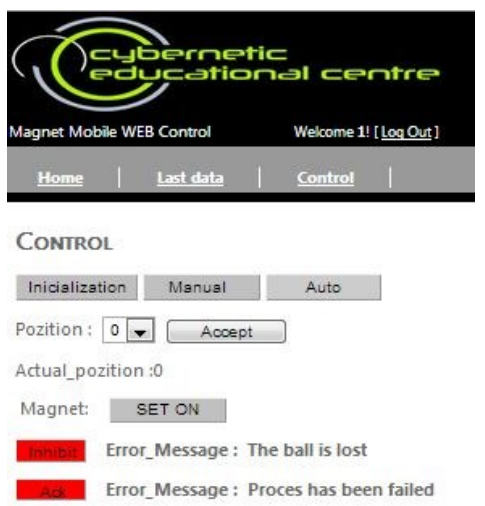

Fig. 4. Mobile web application client for model Magnet

\section{Conclusions}

The main purpose of every industrial company is to build an effective system and reduce its costs. This paper described solutions, benefits and risks of integrating a SCADA/HMI and cloud computing. Our selected solution was introduced as a solution for small industrial companies to store their production data through the SOA service, which is implemented over a realized SCADA/HMI from Wonderware System Platform. The benefit and contribution of this solution is in parallel data processing with a 
SCADA/HMI and the cloud, which doesn't influence the realized SCADA/HMI system, Others benefits are interactivity of the stored data with report analysis tools, web clients, NFS clients and safety of the data. This solution should increase the stability and speed in communication between an industrial company, customers and suppliers.

Acknowledgments. This research was funded by a grant (No. 021TUKE-4/2012) from the KEGA (50\%) and VEGA - 1/0286/11 (50\%).

\section{References}

1. Alcaraz, C., Agudo, I., Nunez, D., Lopez, J.: Managing Incidents in Smart Grids à la Cloud. In: Cloud Computing Technology and Science (CloudCom), 2011 IEEE Third International Conference, pp.527-531 (2011)

2. DeCandia, G., Hastorun, D.,Jampani, M., Kakulapati, G., Lakshman, A., Pilchin, A., Sivasubramanian, S., Vosshall, P., Vogels, W.: Dynamo: amazon's highly available key-value store. In: SOSP '07. New York, NY, USA: ACM, pp. 205-220, (2007)

3. Youseff, L., Butrico, M., Da Silva, D.: Toward a Unified Ontology of Cloud Computing. In: Grid Computing Environments Workshop, 2008, GCE '08, pp.1-10, (2008)

4. Drungilas, D., Bielskis, A. A.: Cloud Interconnected Affect Reward based Automation Ambient Comfort Controller, Electronics and electrical engineering, vol. 18, no. 10, pp. 49-52, (2012)

5. Finn, A., Vredevoort, H., Lownds, P., Flynn, D.: Microsoft Private Cloud Computing Copyright (C) 2012 by John Wiley \& Sons, Inc., Indianapolis, Indiana Published simultaneously in Canada (2012)

6. Alcaraz, C., Fernandez, G., Roman, R., Balastegui, A., Lopez, J.: Secure Management of SCADA Networks. In: New Trends in Network Management, CEPIS by Novática (ATI, Spain), vol. 9, no. 6, pp. 22-28 (2008)

7. Yang, Y., McLaughlin, K., Littler, T., Sezer, S., Im, E. G., Yao, Z.Q., Pranggono, B., Wang, H.F.: Man-in-the-middle attack test-bed investigating cyber-security vulnerabilities in Smart Grid SCADA systems. In: Sustainable Power Generation and Supply (SUPERGEN 2012), International Conference, pp.1-8 (2012)

8. Liguš, J., Zolotová, I., Karch, P., Ligušová, J.: Information and control system of traverse and its integration into cybernetic centre. In: Electronics and electrical engineering, no. 6 (2010), p. 147-152, (2010)

9. Pavlík, M., Mihal', R., Laciňák, L., Zolotová, I.: Supervisory control and data acquisition systems in virtual architecture built via VMware vSphere platform. In: Recent Researches in Circuits and Systems: Proceedings of the 16th WSEAS International Conference on Circuits (part of CSCC '12). Kos Island, Greece, pp. 389-393 (2012)

10. Petersen, K., Spreitzer, M., Terry, D., Theimer, Bayou, M.: Replicated database services for world-wide applications. In EW 7: Proceedings ofthe 7th workshop on ACMSIGOPS European workshop, New York, NY, USA: ACM, pp. 275-280 (1996)

11. Davies, A.: High Availability MySQL Cookbook, Packt Publishing Ltd (2010) 